\title{
COVID-19 vaccine-related functional neurological disorders in the emergency department
}

\author{
Om P. Sanjeev ${ }^{1}\left(\right.$ Alka Verma $^{1} \cdot$ V. E. Mani ${ }^{2} \cdot$ R. K. Singh ${ }^{1}$
}

Received: 5 December 2021 / Accepted: 14 January 2022 / Published online: 2 March 2022

(c) The Author(s), under exclusive licence to Canadian Association of Emergency Physicians (CAEP)/ Association Canadienne de Médecine d'Urgence (ACMU) 2022

Dear Editor,

Functional Neurological Disorder Society in a press note in January' 21 stated that functional neurological disorder is expected to develop in some individuals after COVID-19 vaccination [1]. Heightened stress of pandemic, uncertainty about vaccine's efficacy/safety and transient symptoms/discomfort after vaccination, all are likely precipitating causes. We report two such cases of COVID-19 vaccine-related functional neurological disorder, who visited the emergency department (ED) of a tertiary care centre and were admitted for up to $48 \mathrm{~h}$.

During initial vaccination campaigns in India, two patients reported to our ED with functional symptoms developed after COVID-19 vaccine (Covishield, AstraZeneca, Pune, India) inoculation. The first case, a 42-year-old female, was a health care professional with previous history of mood swings who developed episodic loss of consciousness and unable to open both eyes after first dose of COVID-19 vaccine. Symptoms were non-consistent and she was responding to painful stimuli. However, non-contrast CT scan of brain was obtained to rule out any intracranial pathologies. The second case was a 28 -year-old house-wife

Om P. Sanjeev

opsanjeev@gmail.com

Alka Verma

dralka.verma91@gmail.com

V. E. Mani

vinita.daniel@gmail.com

R. K. Singh

ratenderrks70@gmail.com

1 Department of Emergency Medicine, Sanjay Gandhi Post Graduate Institute of Medical Sciences, House No. 429; Sanskriti EnclaveEldeco Udyan 2; Raebareli Road, Lucknow 226025, India

2 Department of Neurology, Sanjay Gandhi Post Graduate Institute of Medical Sciences, Lucknow, India with previous history of sleep disturbances. She presented to ED with complaints of paraparesis and bilateral foot drops immediately after second dose of vaccine. On neurological examination, findings were inconsistent. Magnetic Resonance Imaging of lumbosacral spine and nerve conduction studies were unremarkable, however carried out to rule out any true deficit. Both patients were managed with proper counselling, oral anxiolytics and were discharged after observation of 22 and $48 \mathrm{~h}$, respectively.

Functional neurological disorders has an incidence of 4-12/100,000 population per year and a prevalence of $50 / 100,000$ population based on a community registry [2] These are common cause of diagnostic concern with expensive, elaborate investigations and poly-pharmacy. The diagnosis is based on eliciting inconsistent clinical signs in the neurological examination, which are not congruent with any disease [3]. In lieu of ongoing COVID-19 pandemic, a crucial concern, however, is fear of misdiagnosis, as side effects and complications are yet to be extensively studied for COVID-19 vaccines. Positive but less reliable diagnostic features of functional neurological disorder include precise midline splitting of vibration sense across the single bones of the forehead or sternum, or a sharply demarcated sensory loss at groin or shoulder [4]. In our cases, all these uncommon clinical signs could not be reliably elicited, however. Side effects and complications related with COVID-19 vaccines were largely unknown, so both patients were admitted for observation and relevant investigations.

Actual number of vaccine-related functional neurological disorders may be more than reported. As the pandemic and vaccination campaigns both are ongoing, more such cases may report to ED. Vaccine safety data can be shared with recipients to allay their anxiety. In case they report to ED, a thorough neurological examination is suggested to curtail any prolongation of disorder. Relevant investigations and imagings can be carried out to rule out any misdiagnosis. 
Funding There is no funding source.

\section{Declarations}

Conflict of interest We declare there is no conflict of interest with any of the authors. We declare we will abide to the rules of Journal after acceptance for copyright transfer.

\section{References}

1. Functional Neurological Disorder Society. Press release from the Functional Neurological Disorder Society. Published January 19;
2021. https://www.fndsociety.org/UserFiles/file/FNDSSocietyPres sReleaseCOVIDVaccines.pdf. Accessed 19 Feb 2021

2. Espay AJ, Aybek S, Carson A, et al. Current concepts in diagnosis and treatment of functional neurological disorders. JAMA Neurol. 2018;75:1132-41.

3. Espay AJ, Lang AE. Phenotype-specific diagnosis of functional (psychogenic) movement disorders. Curr Neurol Neurosci Rep. 2015;15:32.

4. Stone J, Vermeulen M. Functional sensory symptoms. Handb Clin Neurol. 2016;139:271-81. 